\title{
Inteligencia emocional y calidad de vida profesional en el sector industrial
}

\author{
Martin Sanchez-Gomez \\ sanchgom@uji.es \\ Edgar Breso Esteve \\ breso@uji.es
}

\section{Resumen}

Cada vez se tienen más presentes las investigaciones sobre inteligencia emocional (IE) a la hora de analizar factores relacionados con el funcionamiento en las organizaciones. Sin embargo, pocos son los estudios que han tratado de conocer cómo afecta la IE de los altos cargos sobre la salud de los trabajadores a su cargo. Por ello, el presente proyecto pretende realizar una evaluación detallada de la inteligencia emocional de cargos intermedios para conocer su relación con la calidad de vida profesional de los operarios de una empresa industrial a su cargo. La muestra se compone de 181 empleados, con edades comprendidas entre 20 y 61 años (media de edad $=38,8$ ). Los trabajadores fueron evaluados a través de dos cuestionarios: el MEIT-Mobile Emotional Intelligence Test (Sanchez-Gomez y Breso 2019) en su versión para entornos laborales y el CVP-Cuestionario de Vida Profesional (Martín et al. 2004). Los resultados en cuanto a la relación entre la IE de los mandos intermedios y la CVP de los trabajadores de la organización indican correlaciones significativas pero moderadas. En la discusión se plantean aspectos de mejora en futuras investigaciones y distintas líneas de actuación para incorporar la IE en entornos organizacionales.

Palabras clave: inteligencia emocional, calidad de vida profesional, bienestar, industria, supervisores, operarios.

\section{Abstract}

Research on emotional intelligence (EI) is increasingly present when analyzing factors related to the functioning of organizations. However, few studies have tried to know how the El of the high positions affects the health of the workers in their charge. Therefore, this project aims to perform a detailed assessment of the emotional intelligence of intermediate positions to know their relationship with the quality of professional life of the operators of an industrial company under their charge. The sample consists of 181 employees, aged between 20 and 61 years (average age $=38.8$ ). The workers were evaluated through two questionnaires: the MEIT-Mobile Emotional Intelligence Test (Sanchez-Gomez y Breso 2019) in 
its version for work environments and the CVP-Professional Life Questionnaire (Martín et al. 2004). The results regarding the relationship between the El of the middle managers and the CVP of the workers of the organization, indicate significant but moderate correlations. In the discussion, aspects of improvement in future research and different lines of action to incorporate El in organizational settings are considered.

Keywords: emotional intelligence, professional quality of life, welfare, industry, supervisors, workers.

\section{Introducción}

\section{Las emociones en los entornos de trabajo}

La investigación en los últimos años ha demostrado que, además de información y formación reglada, se necesitan otro tipo de habilidades no cognitivas a la hora de facilitar el desarrollo en el ámbito laboral (Moreno-Jiménez, Blanco-Donoso, AguirreCamacho, de Rivas y Herrero 2014). Diversos estudios científicos de prestigio reconocen la importancia de los aspectos emocionales y sociales para facilitar la adaptación de las personas a los cambios (Lopes y Salovey 2004; Mayer y Cobb 2000). En un entorno como el laboral, repleto de cambios constantes, es una variable a tener muy en cuenta, ya que según Castro (2005) es el afrontamiento de estos cambios lo que repercute directamente en la salud física y mental del individuo.

Según Mayer y Salovey (1997) la inteligencia emocional (IE) es una habilidad centrada en el procesamiento de la información emocional, que unifica las emociones y la cognición, permitiendo utilizar nuestras emociones para facilitar un razonamiento más efectivo. Estos autores explican la IE como una habilidad que se divide en cuatro componentes o ramas:

- Percepción emocional. Supone la habilidad para identificar y reconocer tanto los propios sentimientos como los de aquellos que te rodean.

- Facilitación o asimilación emocional. Implica la habilidad para tener en cuenta los sentimientos cuando razonamos o solucionamos problemas.

- Comprensión emocional. Es la habilidad para desglosar el amplio y complejo repertorio de señales emocionales, etiquetar las emociones y reconocer en qué categorías se agrupan los sentimientos.

- Regulación emocional. Aquella capacidad para estar abierto a los sentimientos, tanto positivos como negativos, y reflexionar sobre los mismos para descartar o aprovechar la información que los acompaña en función de su utilidad. Además, incluye la habilidad para regular las emociones propias y ajenas, moderando las emociones negativas e intensificando las positivas.

Wan, Xie y Cui (2016) encontraron asociaciones entre todas las dimensiones de la inteligencia emocional con el afrontamiento productivo. Estos hallazgos defienden que, trabajando la IE, se pueden mejorar diferentes áreas laborales. Además, resulta de especial importancia desarrollar estas capacidades en líderes, porque son las personas que mejor deben saber manejar las emociones en una organización, ya que, sin esta habilidad, no podrían organizar de forma productiva las situaciones cambiantes que surgen en su equipo (Goleman, Boyatzis y McKee 2016). Una parte del éxito de un líder con su equipo está directamente relacionado con la inteligencia emocional y, concretamente, con controlar las propias emociones y potenciar los sentimientos positivos en los equipos de trabajo (Goleman, Boyatzis y McKee 2016). Además, se ha demostrado que la capacidad de los líderes de expresar emociones de forma adecuada tiene relación con el rendimiento de sus subordinados (Dabke 2016). 


\section{Calidad de vida profesional}

La relación entre el mundo emocional y la calidad de vida en la esfera laboral ha sido demostrada por múltiples autores (Fisher 2000; Todorova, Bear y Weingart 2014; Hagemeister y Volmer 2018). Según Martín et al. (2004), la calidad de vida profesional (CVP) puede definirse como la experiencia de bienestar secundaria a la percepción de equilibrio entre las demandas de un trabajo profesional y los recursos disponibles para afrontarlas. Estos autores dividen el constructo CVP en tres dimensiones: apoyo directivo, carga de trabajo y motivación intrínseca (Martín et al. 2004).

Hay varias razones por las cuales existe relación entre la CVP y la IE de los trabajadores. A nivel interpersonal, se espera que la conciencia emocional y los procesos regulatorios asociados con la IE beneficien las relaciones sociales de las personas y, por lo tanto, afecten la experiencia de la emoción y el estrés experimentado en el trabajo. Intrapersonalmente, el uso de la emoción y el estar al tanto de las propias emociones puede conducir a la regulación del estrés y la emoción negativa para que uno pueda desempeñarse mejor en el trabajo (Kafetsios y Zampetakis 2008).

La investigación que ha examinado empíricamente las relaciones entre la IE y la satisfacción en el trabajo ha arrojado resultados diversos. El estudio de Carmeli en 2003 halló relaciones débiles entre las medidas de IE rasgo y la satisfacción laboral en 256 altos directivos, al igual que hicieron Kafetsios y Loumakou (2007) con 475 profesores de educación secundaria. Por el contario, un estudio en un grupo de gerentes encontró fuertes vínculos entre la El y la satisfacción laboral (Lopes, Grewal, Kadis, Gall y Salovey 2006).

Cabe mencionar que en la literatura consultada no se encontraron trabajos que estudien la relación entre la IE de altos cargos o mandos intermedios de una organziación con la CVP de los empleados a su cargo. Por lo tanto, el propósito de este estudio ha sido conocer la relación existente entre estos dos aspectos. Tras analizar la literatura científica previa, esperamos encontrar una relación positiva entre la inteligencia emocional de los líderes de la empresa y la calidad de vida profesional de sus subordinados, en concreto en las dimensiones apoyo directivo y motivación extrínseca. En el caso de la dimensión carga de trabajo tambien se espera encontrar una relación significativa con la IE del líder, aunque en este caso negativa.

\section{Método}

\section{Muestra}

Se han evaluado un total de 208 trabajadores de una empresa dedicada al sector industrial ubicada en la provincia de Alicante, lo que corresponde al $70 \%$ del total de la plantilla. Esta empresa fue seleccionada por conveniencia, debido a la facilidad de acceso. De los 208 trabajadores, 27 (13\%) pertenecen a la categoría de mandos intermedios, mientras que los 181 restantes ( $87 \%$ ) son operarios, los cuales no tienen responsabilidad sobre otros compañeros. Las edades de los mandos intermedios se encuentran entre 26 y 55 años, siendo la edad media de 39,9 (DT = 7,78). El $90 \%$ de los integrantes de este grupo son hombres. Por su parte, las edades de los operarios se encuentran entre 20 y 63 años, siendo la edad media de 34,4 (DT $=5,12$ ). Aquí el porcentaje de hombres es de 80,6 , mientras que el $19,4 \%$ son mujeres. La participación de los trabajadores en las evaluaciones ha sido voluntaria y sin remuneración a cambio. 


\section{Instrumentos}

En primer lugar, evaluamos la calidad de vida profesional mediante el cuestionario CVP-35 en papel (Martín et al. 2004). Este cuestionario está compuesto por 35 ítems divididos en cuatro dimensiones para su corrección: apoyo directivo, cargas de trabajo, motivación intrínseca y calidad de vida profesional global percibida. Las puntuaciones en esta escala van desde el 1 (mínimo) hasta el 10 (máximo). El alfa de Cronbach indica una fiabilidad de 0,82. Esta variable fue medida únicamente en operarios.

Por otro lado, la evaluación de la IE se realizó mediante MEIT (Sanchez-Gomez y Breso 2019), una prueba online que evalúa la percepción emocional (mediante 19 pantallas), la comprensión emocional ( 7 pantallas) y la gestión emocional (14 pantallas). La prueba de fiabilidad de dos mitades indica una fiabilidad de 0,88 . La fiabilidad en las diferentes ramas de percepción, comprensión y gestión osciló entre 0,74 y 0,90 , mientras que en las tareas individuales osciló entre un mínimo de 0,72 y un máximo de 0,91. La IE fue medida solo en mandos intermedios.

\section{Procedimiento}

A la hora de evaluar a la muestra de participantes, se optó por contar, al menos, con 5 operarios por cada supervisor; solo de esta forma podríamos alcanzar unos resultados válidos. La obtención de esta muestra fue de forma aleatoria mediante el orden alfabético del primer apellido. Además, se estimó oportuno eliminar del listado a los trabajadores que llevaban menos de 4 meses en la empresa, ya que estos podían verse afectados por primeras impresiones $u$ opiniones sesgadas.

Para la administración de las pruebas en formato papel, se realizaron grupos de máximo 20 trabajadores, dependiendo de su disponibilidad, para no interferir en el trabajo habitual. De media tardaron unos 40 minutos en su realización, llegando a invertir 1 hora las personas con más dificultades. Para la realización de la prueba de $\mathrm{IE}$, al ser en formato online, se les envió un correo electrónico con la dirección web y la con traseña necesaria para su realización.

\section{Análisis de datos}

Una vez obtenidas las respuestas, se almacenaron los datos para su posterior análisis mediante el programa estadístico SPSS (versión 25.0). Tras ello se obtuvieron correlaciones bivariadas mediante la $r$ de Pearson para conocer la posible relación entre las variables objeto de estudio. El nivel de significación estadística fue del $5 \%$ en todos los casos $(p<0,05)$.

\section{Resultados}

Antes de analizar si existe relación entre la IE y la CVP, presentamos la tabla 1, donde se muestran las correlaciones entre la dimensiones de IE evaluadas por el MEIT. 
Tabla 1

Correlación entre las dimensiones de IE (Pearson)

\begin{tabular}{llll}
\hline Variable & IE Percepción & IE Comprensión & IE Gestión \\
\hline IE Percepción & 1 & & \\
IE Comprensión & $0,28^{*}$ & 1 & \\
IE Gestión & $0,34^{*}$ & $0,29^{*}$ & 1 \\
\hline
\end{tabular}

* La correlación es significativa en el nivel 0,01

Como se puede observar, los resultados indican que los constructos que componen la IE están relacionados de forma significativa y moderada, demostrando así que miden aspectos que tienen relación pero no son idénticos.

\section{Relación entre IE y CVP}

Tras analizar los resultados obtenidos en este estudio, podemos afirmar que están en consonancia con la hipótesis planteada. Existe relación entre la IE y la CVP. En concreto, existe una relación significativa positiva entre las tres variables de IE con el apoyo directivo y la motivación extrínseca, teniendo esta última una correlación algo más débil. En cuanto a la relación de la IE con la carga de trabajo, no se observa ninguna relación significativa. En la tabla 2 podemos observar los resultados arrojados por el análisis de correlaciones entre las tres dimensiones de IE (eje Y) y la CVP (eje $\mathrm{X})$.

Tabla 2

Correlación entre IE y CVP (Pearson)

\begin{tabular}{llll}
\hline Variable & Apoyo directivo & Carga de trabajo & $\begin{array}{l}\text { Motivación } \\
\text { extrínseca }\end{array}$ \\
\hline IE Percepción & $0,18^{*}$ & 0,10 & $0,11^{*}$ \\
IE Comprensión & $0,17^{*}$ & 0,17 & $0,08^{*}$ \\
IE Gestión & $0,20^{*}$ & 0,09 & $0,16^{*}$ \\
\hline
\end{tabular}

* La correlación es significativa en el nivel 0,01

\section{Discusión y conclusiones}

El presente estudio partió del interés por conocer la relación entre la inteligencia emocional y la calidad de vida laboral, concretamente en un sector poco investigado como el industrial. En la introducción teórica se ha desarrollado cómo afecta la IE en la salud psicosocial de los trabajadores, lo cual refuerza la relevancia de nuestra investigación y la importancia de obtener nuevos datos que aporten valor a este importante e inexplorado campo de la investigación.

Analizando los resultados, hemos podido comprobar que la hipótesis planteada era correcta, al menos de forma parcial, ya que existe una relación siginificativa positiva entre la IE del supervisor y dos de las tres dimensiones de la CVP del trabajador a su cargo: motivación extrínseca y apoyo directivo. Estos resultados siguen la línea de investigaciones previas (Carmeli 2003; Lopes, Grewal, Kadis, Gall y Salovey 2006; Kafetsios y Loumakou 2007) en las que se observa una relación significativa entre la IE y el bienestar emocional del trabajador. Sin embargo, no todas las dimensiones de la CVP han demostrado estar relacionadas significativamente con la IE, ya que la carga de trabajo no se relaciona de forma significativa con ninguna otra variable. 
Hipotetizamos que esto puede deberse al instrumento CVP-35, ya que los autores indican en el manuscrito original que no existe relación significativa entre esta dimensión y la CVP. Los resultados hallados en esta investigación pretenden ampliar los conocimientos acerca de la IE en entonos laborales, así como servir como base para consolidar futuras investigaciones. Sin embargo, debemos tener en cuenta la naturaleza del mismo y el muestreo por conveniencia, por lo que los datos deben ser tomados con cautela y su generalización está limitada, pudiendo realizarse solo en muestras similares a la detallada en este trabajo.

Los hallazgos conseguidos en la investigación deben servir para el desarrollo de intervenciones a nivel organizacional que contribuyan al desarrollo de la CVP de los trabajadores. Hasta la fecha, las intervenciones para mejorar la calidad de vida en el trabajo se plantean desde dos enfoques (Sánchez y Breso 2013). Por un lado, aquellas que transforman los procesos o la estructura organizacional (Farughi, Alaniaza y Mousavipour 2014) y, por otro, las que trabajan la capacitación del trabajador (Casañas, Catalán, Raya y Real 2014). Las primeras intervenciones buscan reforzar o reestructurar la organización, lo cual sería muy útil para mejorar la dimensión carga de trabajo, mientras que las segundas incluyen técnicas y estrategias para facilitar y mejorar la conducta de los trabajadores, lo que ayudaría a desarrollar las dimensiones apoyo directivo y motivación intrínseca.

En cuanto a recomendaciones para futuras investigaciones, nos gustaría señalar la importancia de poner énfasis en la confidencialidad de los cuestionarios, ya que, de no hacerlo, los trabajadores son reticentes a su realización, lo cual puede desembocar en respuestas sesgadas y no fiables. Estudios futuros deben tratar de realizar análisis más exhautivos en los que se trabaje con regresiones que puedan explicar si existen relaciones de causalidad entre las variables objeto de estudio. Para finalizar, es importante resaltar que, a pesar de la aparición de múltiples libros, revistas y manuales sobre la importancia de la IE, aún resulta necesario un largo camino por recorrer hasta lograr que las emociones tomen el espacio que merecen en los entornos laborales. Esperamos que este trabajo sume su granito de arena a la causa y forme parte de un número creciente de investigaciones que defienden la importancia de la IE en entornos organizacionales.

\section{Referencias bibliográficas}

Carmeli, Abraham. 2003. "The relationship between emotional intelligence and work attitudes, behavior and outcomes». Journal of Managerial Psychology 18: 788813.

Castro, Rebeca. 2005. «Síndrome de burnout o desgaste profesional. Breve referencia a sus manifestaciones en profesores universitarios». Anuario 28: 159-184.

Casañas, Rocío, Rosa Catalán, Antonia Raya y Jordi Real. 2014. «Efectividad de un programa grupal psicoeducativo para la depresión mayor en atención primaria: ensayo clínico controlado aleatorizado». Revista de la Asociación Española de Neuropsiquiatria 34(121): 145-146.

Dabke, Deepika. 2016. «Impact of leader's emotional intelligence and transformational behavior on perceived leadership effectiveness: A multiple source view». Business Perspectives and Research 4(1): 27-40.

Farughi, Hiwa, Seiran Alaniaza y Seyed Hamed Mousavipour. 2014. «Presenting a Framework of Reengineering methodology for Organizational Diagnosis and Process Improvement (Case Study: Industrial Estate Company of Kurdistan)». International Journal of Management, Accounting and Economics 1(4): 295-310. 
Fisher, Cynthia D. 2000. «Mood and emotions while working: missing pieces of job satisfaction?». Journal of organizational behavior 21(2): 185-202.

Goleman, Daniel, Richard Boyatzis y An McKee. 2016. El líder resonante crea más: El poder de la inteligencia emocional. Barcelona: Penguin Random House.

Hagemeister, Alexandra y Judith Volmer. 2018. «Do social conflicts at work affect employees' job satisfaction? The moderating role of emotion regulation». International Journal of Conflict Management 29(2): 213-235.

Kafetsios, Konstantinos y Mary Loumakou. 2007. "A comparative evaluation of the effects of trait emotional intelligence and emotion regulation on affect at work and job satisfaction». International Journal of Work Organization and Emotion 2(1): 71-87.

Kafetsios, Konstantinos y Leonidas A. Zampetakis. 2008. «Emotional intelligence and job satisfaction: Testing the mediatory role of positive and negative affect at work». Personality and individual differences 44(3): 712-722.

Lopes, Paulo N. y Peter Salovey. 2004. «Toward a broader education: Social, emotional, and practical skills». En Building academic success on social and emotional learning, ed. Joseph E. Zins, Roger P. Weissberg, Margaret C. Wang y Herbert J. Walberg (76-93). New York: Teachers College Press.

Lopes, Paulo N., Daisy Grewal, Jessica Kadis, Michelle Gall y Peter Salovey. 2006. «Evidence that emotional intelligence is related to job performance and affect and attitudes at work». Psicothema 18: 132-128.

Martín, Jesús, José Alfonso Cortés, Manuel Morente, Marcial Caboblanco, Javier Garijo y Alberto Rodríguez Balo. 2004. «Características métricas del cuestionario de calidad de vida profesional (CVP-35)». Gaceta Sanitaria 18(2): 129-136.

Mayer, John y Peter Salovey. 1997. "What is emotional intelligence?». En Emotional Development and Emotional Intelligence: Implications for Educators, ed. Peter Salovey y David Sluyter (3-31). New York: Basic Books.

Mayer, John y Casey D. Cobb. 2000. «Educational Policy on Emotional Intelligence: Does It Make Sense?». Educational Psychology Review 12(2): 163-183.

Moreno-Jiménez, Bernardo, Luis M. Blanco-Donoso, Aldo Aguirre-Camacho, Sara de Rivas y Marta Herrero. 2014. "Habilidades sociales para las nuevas organizaciones». Behavioral Psychology/Psicología Conductual 22(3): 587604.

Sánchez, Juan Pedro y Edgar Breso. 2013. «Cómo desarrollar las competencias emocionales en directivos y supervisores». Gestión Práctica de Riesgos Laborales 110: 22-26.

Sanchez-Gomez, Martin y Edgar Breso. 2019. «The Mobile Emotional Intelligence Test (MEIT): An Ability Test to Assess Emotional Intelligence at Work». Sustainability 11(3): 827. https://doi.org/10.3390/su11030827

Todorova, Gergana, Julia Bear y Laurie R. Weingart. 2014. "Can conflict be energizing? A study of task conflict, positive emotions, and job satisfaction». Journal of Applied Psychology 99(3): 451.

Wang, Yefei, Guangrong Xie y Xilong Cui. 2016. «Effects of emotional intelligence and self-leadership of students coping with stress». Social Behavior and Personality 44: 853-864. 